\title{
Clinical utility of genetic testing in the early diagnosis of Danon disease mimicking hypertrophic cardiomyopathy: a case report
}

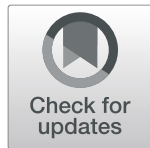

\author{
Valeria Novelli, ${ }^{1,2+}$, Antonio Bisignani ${ }^{3,4+}$, Gemma Pelargonio ${ }^{3,4}$, Guido Primiano ${ }^{5,6}$, Maria Lucia Narducci ${ }^{3,4}$, \\ Vincenzo Palmieri ${ }^{7}$, Francesco Danilo Tiziano ${ }^{1,2}$, Paolo Zeppilli ${ }^{7}$, Serenella Servidei ${ }^{5,6}$, Filippo Crea ${ }^{3,4}$ and \\ Maurizio Genuardi ${ }^{1,2^{*}}$
}

\begin{abstract}
Background: Danon disease (OMIM 300257) is an X-linked lysosomal storage disorder, characterized by hypertrophic cardiomyopathy (HCM), skeletal myopathy, variable intellectual disability, and other minor clinical features. This condition accounts for $\sim 4 \%$ of HCM patients, with a more severe and early onset phenotype in males, causing sudden cardiac death (SCD) in the first three decades of life.

Genetic alterations in the LAMP2 gene are the main cause of this inherited fatal condition. Up to date, more than 100 different pathogenic variants have been reported in the literature. However, the majority of cases are misdiagnosed as HCM or have a delay in the diagnosis.

Case presentation: Here, we describe a young boy with an early diagnosis of HCM. After 2 episodes of ventricular fibrillation within 2 years, genetic testing identified a novel LAMP2 pathogenic variant. Subsequently, further clinical evaluations showing muscle weakness and mild intellectual disability confirmed the diagnosis of Danon disease.

Conclusions: This report highlights the role of genetic testing in the rapid diagnosis of Danon disease, underscoring the need to routinely consider the inclusion of LAMP2 gene in the genetic screening for HCM, since an early diagnosis of Danon disease in patients with a phenotype mimicking HCM is essential to plan appropriate treatment, ie cardiac transplantation.
\end{abstract}

Keywords: Danon disease, Genetic testing, Pathogenic variants, Hypertrophic cardiomyopathy, Phenocopy

\section{Background}

Danon disease (DD; OMIM 300257), also named glycogen storage disease type IIb, is an X-linked lysosomal storage disorder, characterized by hypertrophic cardiomyopathy $(\mathrm{HCM})$, skeletal myopathy, variable intellectual disability and retinal disease [1]. This condition accounts for $\sim 4 \%$ of HCM patients [2], affecting both

\footnotetext{
* Correspondence: maurizio.genuardi@unicatt.it

Valeria Novelli and Antonio Bisignani equally contributed to this work

${ }^{1}$ Fondazione Policlinico Universitario A. Gemelli IRCCS, UOC Genetica Medica, Rome, Italy

${ }^{2}$ Istituto di Medicina Genomica, Università del Sacro Cuore, L.go F. Vito 1, 00168 Rome, Italy

Full list of author information is available at the end of the article
}

genders, with a more severe and early onset phenotype in males. Usually, these present HCM at puberty and require heart transplantation around $18-19$ years of age, while female carriers develop DCM (dilated cardiomyopathy) or HCM during adulthood often without skeletal muscle involvement [2]. Also, left-ventricular noncompaction phenotype has been reported in a patient affected by Danon disease [3].

Alterations in the LAMP2 gene, coding for the lysosome-associated membrane protein 2 , have been identified as the main cause of Danon disease. So far, more than 100 different pathogenic variants involving the LAMP2 locus have been described,

(c) The Author(s). 2020 Open Access This article is licensed under a Creative Commons Attribution 4.0 International License, which permits use, sharing, adaptation, distribution and reproduction in any medium or format, as long as you give appropriate credit to the original author(s) and the source, provide a link to the Creative Commons licence, and indicate if changes were made. The images or other third party material in this article are included in the article's Creative Commons licence, unless indicated otherwise in a credit line to the material. If material is not included in the article's Creative Commons licence and your intended use is not permitted by statutory regulation or exceeds the permitted use, you will need to obtain permission directly from the copyright holder. To view a copy of this licence, visit http://creativecommons.org/licenses/by/4.0/. The Creative Commons Public Domain Dedication waiver (http://creativecommons.org/publicdomain/zero/1.0/) applies to the data made available in this article, unless otherwise stated in a credit line to the data. 
including non-synonymous variants as well as microdeletions at Xq24 [4]. Here we report on a young male patient, with an initial clinical misdiagnosis of HCM. This case highlights the pivotal role of genetic testing in the rapid diagnosis of this fatal disease.

\section{Case presentation}

A 16-year-old Italian male, with a family history of sudden cardiac death (SCD), was referred to our center with a diagnosis of HCM. His mother died of suspected postpartum DCM when she was 24 years old. The proband had received a diagnosis of HCM one year before, when he was admitted for palpitation in a secondary hospital. The ECG showed atrial tachycardia. He underwent two unsuccessful right atrial catheter ablations. Electrophysiological workup was negative for the induction of ventricular arrhythmias. Therefore, he was not considered suitable for ICD implantation at that time.

Upon admission to our institution, at the age of 17 years, he was symptomatic for palpitations, with evidence of atrial tachycardia (atrial cycle length $330 \mathrm{msec}$ ) during ECG monitoring, (NYHA class I) (Fig. 1). The echocardiogram confirmed the severe biventricular hypertrophy (LV septum thickness was $30 \mathrm{~mm}$, PWT 23 $\mathrm{mm}$ and LVOT $30 \mathrm{mmHg}$ ), with mild LV outflow tract obstruction, previously documented. Moderate biventricular contractile dysfunction was observed, without any evidence of dilation (Fig. 2a-b). Cardiac MRI was performed and confirmed preserved ventricular volumes associated with a severe increase in RV and LV wall thickness and moderate biventricular dysfunction (LVEF 45\%; RVEF 42\%). The calculated LV mass was $268 \mathrm{~g} / \mathrm{m}^{2}$. T2w sequences indicated multiple "patchy" areas of increased signal intensity within the biventricular wall, associated with hypoperfusion at rest and late gadolinium enhancement, suggesting multiple foci of fibrosis.

Due to the persistence of symptomatic atrial tachycardia, 3D electroanatomic mapping of atrial tachycardia activation was performed. The earliest atrial activation was recorded close to the area between right pulmonary vein. Subsequently, antral right pulmonary veinelectrical isolation was performed by Smarttouch Navistar catheter (Biosense Webster) (max temp $32^{\circ} \mathrm{C}$, max power $30 \mathrm{~W}$ ) with interruption of clinical tachycardia. Atrial tachycardia was not re-inducible any longer up to 4 extra-stimuli.

Drug therapy during hospitalization included betablocker (metoprololo $100 \mathrm{mg}$ ), diuretic (furosemide12.5 $\mathrm{mg}$ ) and anticoagulant therapy for 3 months after catheter ablation. Upon admission, genetic counseling was offered to the family and genetic testing was performed (Fig. 3).

Subsequently, further neurological investigations, including skeletal muscle biopsy were performed during this period. These revealed mild intellectual disability, mild weakness of distal upper and lower limb muscles and bilateral pes cavus, with an elevated serum CK (562 UI/L). In particular, clinical history revealed intellectual and adaptive functioning deficits in conceptual, social, and

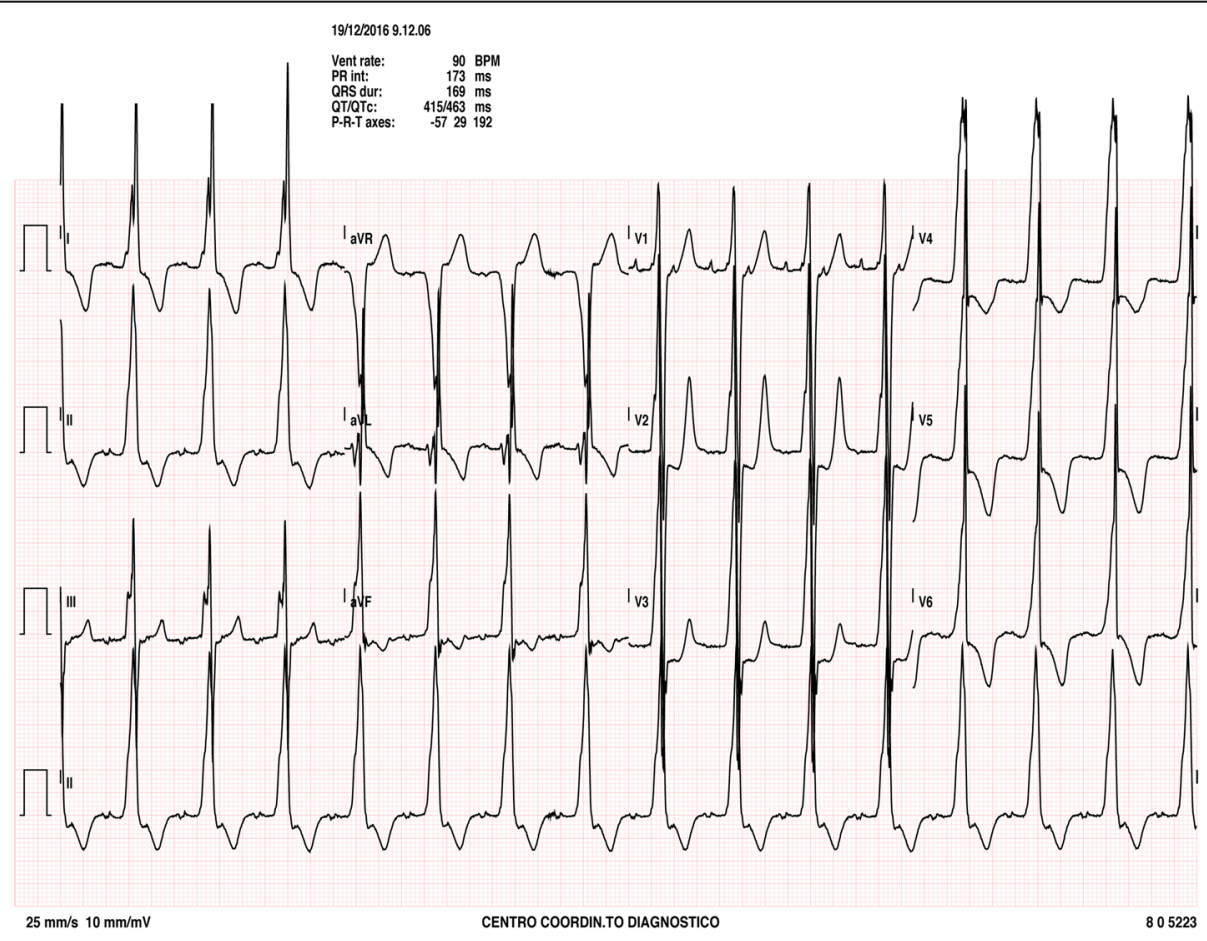

Fig. 1 ECG at admission shows atrial tachycardia with 2:1 AV block 

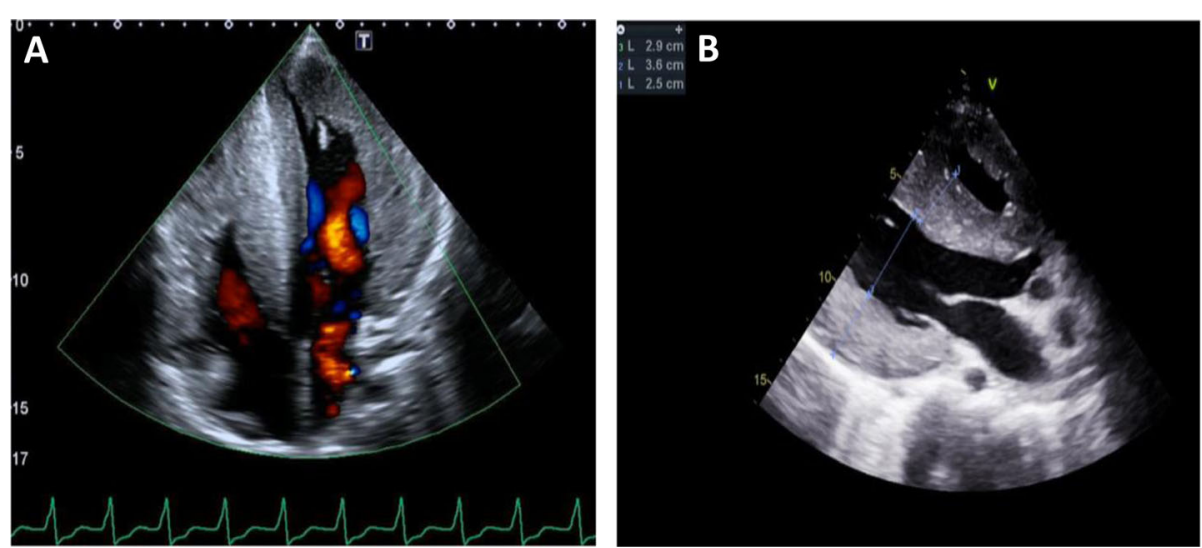

Fig. 2 a-b Echocardiogram showing apical four chamber (A) and parasternal long-axis (B) views showing severe biventricular hypertrophy with a maximum thickness of $30 \mathrm{~mm}$

practical domains with childhood onset, including difficulties in acquiring academic skills, immature language and the need of assistance in daily living tasks. No other clinical exams was conducted at that time. Finally, since the predicted 5 years risk of SCD was $4.34 \%$, according to the 2014 guidelines on diagnosis and management of HCM, ICD was implanted and muscle biopsy was requested.

\section{Genetic testing}

DNA isolated from peripheral blood leukocytes was screened for genes associated with HCM and related phenotypes using a sequencing panel (Custom Ion Panel, ThermoFisher-US) including 11 genes (MYBPC3,

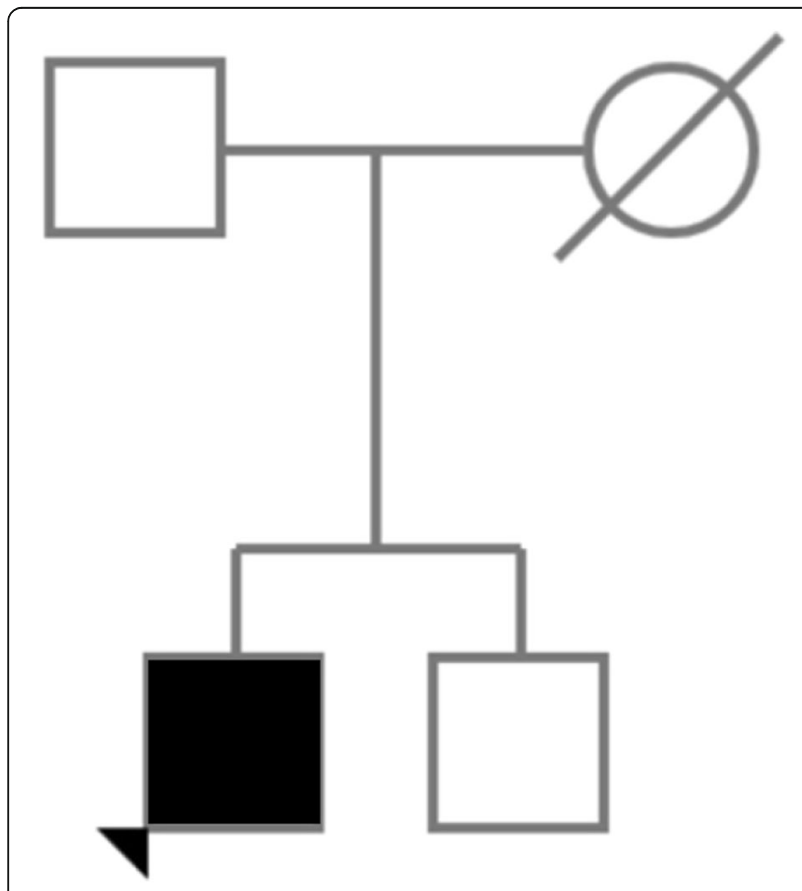

Fig. 3 Pedigree of the family
MYH7, TNNT2, TNNI3, TPM1, MYL2, MYL3, ACTC1, $P L N, L A M P 2, G L A)$, encoding for sarcomeric and non sarcomeric proteins. All deep intronic and synonymous coding variants were excluded from further analysis. Then, we filtered out all the variants with an allelic frequency (MAF) of more than $1 \times 10^{-4}$ in (gnomad.broadinstitute.org). Variant annotation results showed a hemizygous $1 \mathrm{bp}$ deletion, c.453delT, in LAMP2 (NM 002294), which was confirmed by Sanger sequencing. The variant (chrX:119582932_delT) causes a frameshift in exon 4, p.(F151Lfs"32) resulting in a putative premature stop codon at position 183 of the protein. To the best of our knowledge, it has not previously been reported in the literature, and it is absent in gnomAD and in other electronic databases, including ClinVar (ncbi.nlm.nih.gov/clinvar). Clinical variant interpretation was performed using the ACMG criteria [5]. In particular, the variant was classified as likely pathogenic based on the molecular defect (PVS1; frameshift with premature protein truncation) and on its allele frequency in the general population (PM2).

At that time, genetic counseling was also offered to the older brother of the patient. During counseling, it was explained that probably the mother was a carrier of the LAMP2 pathogenic variant, and that he had a 50\% chance of having inherited it. He then agreed to undergo targeted genetic testing for the disease causing variant. The result was negative, indicating that he is not at risk of developing manifestations of Danon disease.

\section{Muscle biopsy}

Skeletal muscle biopsy (Fig. 4) demonstrated mild variation in fiber size and numerous scattered small dot-like basophilic granules and vacuoles that showed an enhanced activity of lysosomal acid phosphatase and acetylcholine esterase. The lysosomal vacuoles had the characteristics of autophagic vacuoles with unique sarcolemma features since the limiting membranes were positive for 


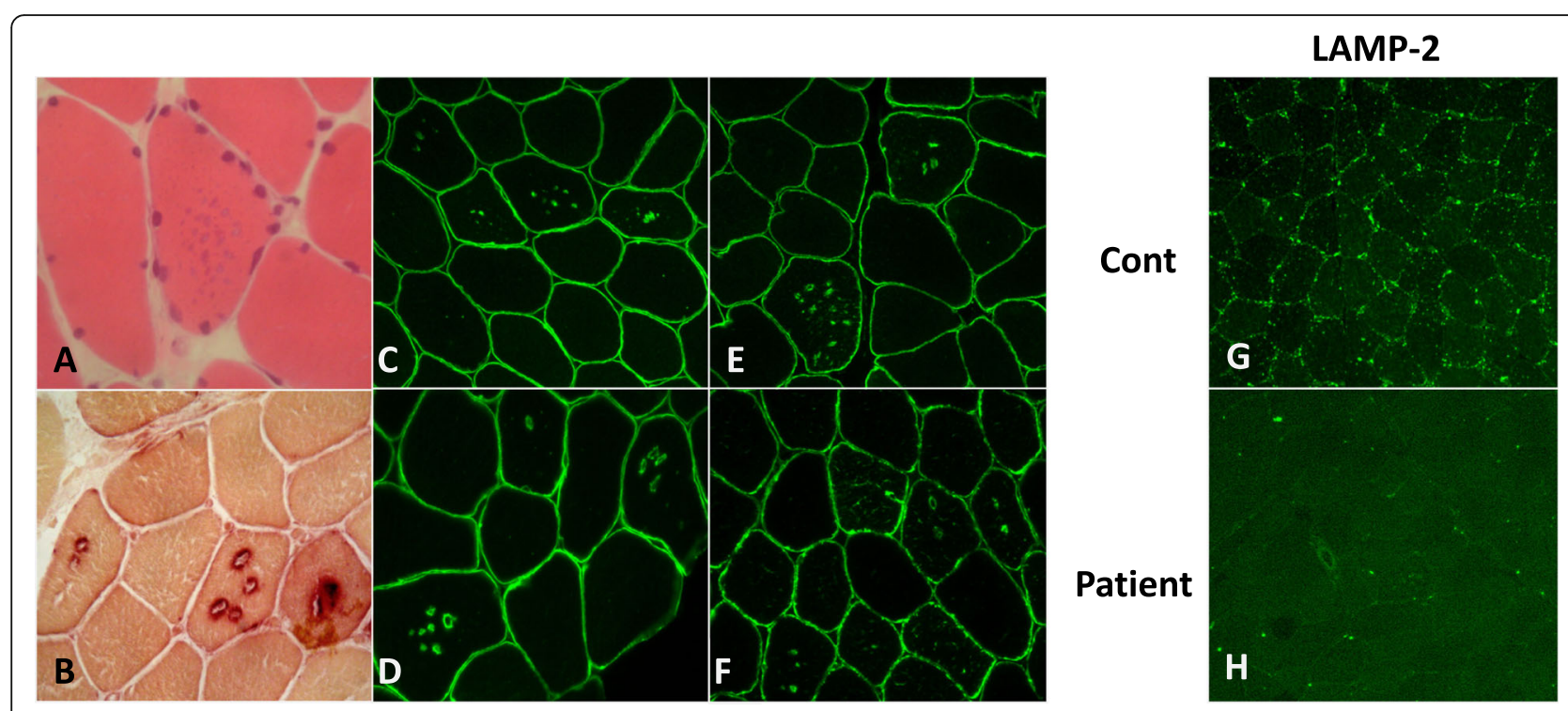

Fig. 4 a-b-c-d-e-f-g-h: Muscle pathology: hematoxylin and eosin staining a shows small basophilic granular structures in the muscle fibers, which are acetylcholine esterase $\mathbf{b}$ positive autophagic vacuoles; immunohistochemistry using dystrophin $\mathbf{c}, \beta$-sarcoglycan $\mathbf{d}$, caveolin-3, and a -dystroglycan $\mathbf{f}$ antibodies demonstrates that the limiting membranes of the vacuoles have sarcolemmal features; immunohistochemical staining with anti-LAMP2 antibody $\mathbf{g}$ shows that LAMP-2 expression is present in anunaffected individuaL $\mathbf{h}$ while is absent in skeletal muscles of the patient

sarcolemma proteins such as dystrophin, sarcoglycans, dystroglycans, and caveolin. Immunohistochemistry using anti-lysosome-associated membrane protein 2 (LAMP2) antibodies revealed the absence of the protein in all fibers, concordant with a diagnosis of Danon disease.

\section{Discussion and conclusions}

The case presented herein demonstrates the role of genetic testing in the early diagnosis of Danon disease. A novel $L A M P 2$ likely pathogenic variant was detected in the proband before a diagnosis of Danon disease was established on clinical grounds.

The natural history of this inherited condition is characterized by a high rate of mortality within the secondthird decade of life. Males, in particular, present a rapid progression of HCM with a mean age at death of 19 years $( \pm 6$ y). In contrast, females can develop either DCM or HCM with a more protracted course, with a mean age at death of 40 years $( \pm 7 \mathrm{y})$.

Due to the rapid progression of this lethal disease, early diagnosis is crucial. However, due to the cardiac phenotype that mimics HCM and often obscures the other clinical manifestations, often present in a mild form, early diagnosis can be challenging, in particular in the pediatric population, when the involvement of other organs may not be evident yet. For these reasons, a strategy for a rapid genetic diagnosis is critical to identify patients at risk.

Next generation sequencing analysis using gene panels offers the possibility of investigating simultaneously a large number of genes involved in a genetically heterogeneous condition at affordable costs and with rapid turnaround time. However, the use of larger panels for the systematic screening of $\mathrm{HCM}$ offers limited additional sensitivity resulting in an increasing number of variants of uncertain significance (VUS). On the other hand, limiting the analysis to genes associated with non-syndromic HCM, ie sarcomeric genes, might lead to missed or delayed diagnosis in patients affected with syndromic forms of HCM who do not have prominent extracardiac manifestations.

Recently, Alfares et al. [6] analyzing a cohort of 2912 non-syndromic HCM patients, identified 6 cases carrying different $L A M P 2$ pathogenic variants. These results indicate that Danon disease mimicking an isolated HCM phenotype may be difficult to diagnose on a clinical basis alone.

Furthermore, recent data have shown that inclusion of genes associated with non-syndromic HCM diseases, including $L A M P 2$, increases the diagnostic yield of systematic genetic screening for HCM. Analyzing a cohort of 1198 probands with a diagnosis of HCM, it has been observed that $L A M P 2$ has the same utility of a sarcomeric gene, showing a high frequency of rare pathogenic and likely pathogenic variants in the patient population compared to general population databases, ie ExAC [7].

These data confirm that the inclusion of genes associated with HCM syndromic forms diseases in HCM NGS panels can enable early diagnosis of Danon disease, improves the management of these patients, and allows to identify family members at risk of this fatal condition. Indeed, in this case, after the genetic diagnosis of Danon disease, the patient was immediately placed on a waiting list for heart transplant. 
In conclusion, this case underscores the need to routinely analyze the $L A M P 2$ gene as part of the genetic screening for $\mathrm{HCM}$ to assess early diagnosis of Danon disease in patients with a phenotype mimicking isolated HCM. Since in some patients the molecular defect is a large deletion, CNV analysis should also be systematically performed.

\section{Abbreviations}

ACMG: American College of Medical Genetics and Genomics; VUS: Variant of uncertain significance; ExAC: Exome aggregation consortium; HCM: Hypertrophic Cardiomypathy; DCM: Dilated cardiomyopathy; CNV: Copy number variation; SCD: Sudden cardiac death; VF: Ventricular fibrillation; ICD: Implanted cardioverter defibrillator; ECG: Electrocardiogram; LV: Left ventricle; MRI: Magnetic resonance imaging; LGE: Late gadolinium enhancementinsert

\section{Acknowledgments}

Not applicable.

\section{Authors' contributions}

NV and MG: design and coordination of the study. NV, BA: writing, data collection, analysis and interpretation of data. NV, MG, BA: patient's attention, idea, critical revision. BA, GPe, FTD, MLN, FC, GPr and SS: analysis and interpretation of patient's data, design, writing, project management, analysis and interpretation of data. FC, SS, PZ and MG: critical revision. All authors read and approved the final manuscript.

\section{Funding}

Genetic analysis was supported by "Centro Benito Stirpe per la Prevenzione della Morte Improvvisa nel Giovane Atleta".

\section{Availability of data and materials}

All data generated or analysed during this study are included in this published article.

\section{Ethics approval and consent to participate}

All procedures performed in this research were in accordance with the ethical standards of the 1964 Helsinki declaration. All participants in this report voluntarily agreed to participate and signed written consent. The module of consent for diagnostic genetic testing, including all the info regarding the application of the identified data also for research scope, has been approved by the Legal Department of our Institution.

\section{Consent for publication}

A written patient consent for publication, including images and other clinical information has been signed by the father of the proband.

\section{Competing interests}

The authors declare they have no competing interest.

\section{Author details}

${ }^{1}$ Fondazione Policlinico Universitario A. Gemelli IRCCS, UOC Genetica Medica, Rome, Italy. ${ }^{2}$ Istituto di Medicina Genomica, Università del Sacro Cuore, L.go F. Vito 1, 00168 Rome, Italy. ${ }^{3}$ Fondazione Policlinico Universitario A. Gemelli IRCCS, Dipartimento di Scienze Cardiovascolari e Toraciche, Rome, Italy. ${ }^{4}$ Università Cattolica del Sacro Cuore, Rome, Italy. ${ }^{5}$ Fondazione Policlinico Universitario A. Gemelli IRCCS, UOC Neurofisiopatologia, Rome, Italy. ${ }^{6}$ Istituto di Neurologia, Università Cattolica del Sacro Cuore, Rome, Italy. ${ }^{7}$ Fondazione Policlinico Universitario A. Gemelli, IRCCS, Unità di Medicina dello Sport, Università Cattolica del Sacro Cuore, Rome, Italy.

Received: 27 August 2019 Accepted: 9 March 2020

Published online: 05 April 2020

\section{References}

1. Danon MJ, Oh SJ, DiMauro S, Manaligod JR, Eastwood A, Naidu S, Schliselfeld LH. Lysosomal glycogen storage disease with normal acid maltase. Neurology. 1981;31:51-7.

2. Yang Z, McMahon CJ, Smith LR, Bersola J, Adesina AM, Breinholt JP, Kearney DL, Dreyer WJ, Denfield SW, Price JF, et al. Danon disease as an underrecognized cause of hypertrophic cardiomyopathy in children. Circulation. 2005;1121:612-7.

3. Van Der Starre P, Deuse T, Pritts C, Brun C, Vogel H, Oyer P. Late profound muscle weakness following heart transplantation due to Danon disease. MuscleNerve. 2013;47:135-7.

4. Yang Z, Funke BH, Cripe LH, Vick GW 3rd, Mancini-Dinardo D, Peña LS, Kanter RJ, Wong B, Westerfield BH, Varela JJ, et al. LAMP2 microdeletions in patients with Danon disease. Circ Cardiovasc Genet. 2010;3:129-37.

5. Richards S, Aziz N, Bale S, Bick D, Das S, Gastier-Foster J, Grody WW, Hegde $M, L y o n E$, Spector $E$, et al. Standards and guidelines for the interpretation of sequence variants: a joint consensus recommendation of the American College of Medical Genetics and Genomics and the Association for Molecular Pathology. Genet Med. 2015;17:405-24.

6. Alfares AA, Kelly MA, McDermott G, Funke BH, Lebo MS, Baxter SB, Shen J, McLaughlin HM, Clark EH, et al. Results of clinical genetic testing of 2,912 probands with hypertrophic cardiomyopathy: expanded panels offer limited additional sensitivity. Genet Med. 2015;17:880-8.

7. Mazzarotto F, Girolami F, Boschi B, Barlocco F, Tomberli A, Baldini K, Coppini R, Tanini I, Bardi S, Contini E, et al. Defining the diagnostic effectiveness of genes for inclusion in panels: the experience of two decades of genetic testing for hypertrophic cardiomyopathy at a single center. Genet Med. 2019:21:284-92

\section{Publisher's Note}

Springer Nature remains neutral with regard to jurisdictional claims in published maps and institutional affiliations.
Ready to submit your research? Choose BMC and benefit from:

- fast, convenient online submission

- thorough peer review by experienced researchers in your field

- rapid publication on acceptance

- support for research data, including large and complex data types

- gold Open Access which fosters wider collaboration and increased citations

- maximum visibility for your research: over $100 \mathrm{M}$ website views per year

At $B M C$, research is always in progress.

Learn more biomedcentral.com/submission 